\section{Doenças crônicas não transmissíveis: desempenho no cuidado médico em atenção primária à saúde no sul do Brasil}

\author{
Non-communicable chronic diseases: \\ performance of medical care in Primary Health \\ Care in southern Brazil
}

\author{
1 Universidade Federal de \\ Pelotas, Pelotas, Brasil. \\ Correspondência \\ M. Capilheira \\ Universidade Federal de \\ Pelotas. \\ Rua Marechal Deodoro 1160, \\ 3 a andar, Pelotas, $R S$ \\ 96020-220, Brasil. \\ capilheira@brturbo.com.br
}

\begin{abstract}
A cross-sectional study was performed to assess knowledge and performance on the prevention of risk factors for chronic non-communicable diseases (CNCD) in adults, among primary care physicians in the urban health system in Pelotas, Rio Grande do Sul State, Brazil. The study included observation of 422 consultations by 61 physicians. $58.8 \%$ of the patient consultations for CNCD and 25.3\% of those for other reasons lacked any preventive recommendations and/or measurement of weight, height, and blood pressure. For patients with CNCD, the most frequent recommendations were weight loss, more physical activity, and reduced fat and salt intake. Among patients without CNCD, only $39.1 \%$ received any recommendations related to primary prevention. Physician performance for the prevention of CNCD was poor, and focused mainly on tertiary prevention (counseling patients who were already diagnosed with CNCD) as compared to primary prevention (preventing the incidence of CNCD).
\end{abstract}

Chronic Disease; Primary Health Care; Health Services
Marcelo Capilheira 1

Iná S. Santos 1

\section{Introdução}

Estratégias para mudança de estilos de vida na população em geral, visando redução da prevalência de fatores de risco para doenças crônicas não transmissíveis (DCNT), têm sido discutidas e incentivadas em vários países 1,2,3,4,5. Diversas intervenções para reduzir o tabagismo e promover atividade física e consumo de alimentos saudáveis foram realizadas, mas suas avaliações demonstraram resultados discretos 2,4,6. A extensa cadeia causal desde a implantação das intervenções até o impacto, o funcionamento do sistema de saúde (acesso, disponibilidade de exames complementares e medicamentos), o meio ambiente e as características sociodemográficas, culturais e epidemiológicas da população estão entre as razões da baixa efetividade 7 . Alguns autores apontam o envolvimento dos serviços de saúde como uma estratégia importante para o sucesso e sustentabilidade das intervenções preventivas em DCNT 1,2,3,4,5.

Em 2002, a Organização Pan-Americana da Saúde (OPAS) sistematizou um conjunto de recomendações que objetivam prevenir e controlar as DCNT nas Américas. Tal estratégia, conhecida como Iniciativa CARMEN (Conjunto de Acciones para la Reducción Multifactorial de Enfermedades No Transmisibles), intenta influenciar o processo saúde-doença como um todo, melhorando o estado de saúde das populações e reduzindo fatores de risco associados ao de- 
senvolvimento de DCNT ${ }^{3}$. A Iniciativa CARMEN aponta o envolvimento dos serviços e profissionais de saúde como uma ferramenta efetiva e sustentável de intervenção nas comunidades, especialmente na prevenção das DCNT pelo combate aos seus fatores de risco 1,2,3,4,5. Nesse contexto, deve-se ressaltar que o sistema de saúde brasileiro (Sistema Único de Saúde - SUS) oferece como porta de entrada uma rede de serviços (Atenção Primária à Saúde) que se caracteriza pela abordagem preventiva como uma de suas prioridades.

Assim, o envolvimento da Atenção Primária à Saúde na estratégia proposta pela Iniciativa CARMEN é de grande importância. Deve-se conhecer o perfil dos profissionais desse nível de atenção e sua atuação junto aos usuários, a fim de que se planejem estratégias de combate às DCNT adequadas à realidade de cada local. Sendo os recursos humanos elementos centrais da qualidade do cuidado à saúde oferecido à população, o atual estudo consistiu em uma avaliação da estrutura (características dos recursos humanos) e processo de atenção nas unidades básicas de saúde (UBS) de Pelotas, Rio Grande do Sul, Brasil 8. Com essa avaliação, busca-se contribuir para a elucidação do potencial papel dos serviços de Atenção Primária à Saúde, na efetividade de ações para prevenção de DCNT.

O objetivo principal foi avaliar o conhecimento e o desempenho dos médicos atuantes na rede de Atenção Primária à Saúde da cidade de Pelotas, quanto à prevenção e manejo de fatores de risco para DCNT em adultos.

\section{Metodologia}

Conduziu-se um estudo transversal com todos os médicos atuantes nas UBS urbanas de Pelotas que prestassem atendimento clínico a indivíduos de 20 anos de idade ou mais. Foram excluídos os que atendiam exclusivamente as áreas da pediatria e ginecologia. Sete UBS urbanas foram excluídas do estudo: cinco por serem unidades-escola e duas porque o médico já havia sido contatado em outra UBS. O município está localizado no extremo sul do Brasil, apresentando cerca de 340 mil habitantes, sendo 93\% moradores da zona urbana. A rede de Atenção Primária à Saúde da cidade conta com 50 UBS, sendo 35 localizadas na zona urbana, das quais nove contam com a Estratégia Saúde da Família (ESF).

A coleta de dados desenvolveu-se em duas etapas. A primeira etapa consistiu na realização de observações de todos os tipos de consultas de pacientes adultos, em um turno de um dia típico de trabalho dos médicos, que desconheciam os objetivos do estudo. Considerou-se "dia típico" aquele que não ocorreu às vésperas ou imediatamente após os feriados nacionais, regionais ou municipais, nem em dias com manifestações climáticas mais extremas. As observações foram feitas por sete estudantes de medicina, devidamente treinadas, que permaneciam no consultório e registravam em uma lista os itens sobre prevenção e/ou tratamento de doenças cardiovasculares. Como indicadores de processo, registrou-se qualquer investigação sobre fatores de risco, seja pergunta ou comentário do médico ou mencionado espontaneamente pelo próprio paciente. Foram anotadas quaisquer orientações gerais ou específicas dadas pelo médico quanto a nove itens: redução do consumo de sal, redução do consumo de gorduras, adequação do consumo de verduras e frutas, orientação para perder peso (incluía quaisquer orientações, desde redução de alimentos específicos, como batata, massa, refrigerantes, doces, entre outros, até encaminhamento para consulta com nutricionista ou recomendação para manter uma dieta já prescrita), realização de atividade física, cessação do tabagismo e aferição do peso, altura e pressão arterial. Essas medidas de exame físico podiam ser obtidas pelo próprio médico ou outro profissional da equipe. Os itens observados foram baseados em recomendações do Ministério da Saúde do Brasil ${ }^{9}$ e livro-texto específico da Atenção Primária à Saúde 10 .

A queixa principal da consulta foi registrada, sendo posteriormente categorizada como DCNT (hipertensão arterial, diabetes, tabagismo, sedentarismo, obesidade, dislipidemia, sequela de acidente vascular cerebral) e não DCNT.

Após as observações, o prontuário de cada paciente era revisado para identificar se, nos 12 meses anteriores à consulta observada, havia registro médico de DCNT (hipertensão arterial sistêmica, diabetes mellitus, excesso de peso obesidade/sobrepeso - dislipidemia, cardiopatia isquêmica, insuficiência cardíaca, tabagismo e sedentarismo, além de medidas de peso, altura e pressão arterial). Os dados foram extraídos para um formulário especialmente elaborado para o estudo. Os observadores eram orientados a não mostrarem a planilha aos médicos, tampouco comentarem o conteúdo das observações.

A segundo etapa iniciou imediatamente após o término da primeira e consistiu na caracterização dos médicos e na avaliação do conhecimento sobre fatores de risco para DCNT e prevenção de doenças cardiovasculares (indicadores de estrutura). Para essa fase do estudo, foi usado um questionário estruturado autoaplicado pelos médicos. Duas entrevistadoras treinadas conduziram a segunda visita aos médicos. 
Para a caracterização dos médicos, foram coletadas informações demográficas, sobre seu estado de saúde, formação profissional e características de trabalho, incluindo as dificuldades percebidas para o trabalho na UBS. Foram investigadas também as barreiras para a recomendação de práticas preventivas em Atenção Primária à Saúde. Todas as informações foram autorreferidas. O conhecimento dos médicos foi avaliado pela aplicação de sete perguntas do tipo verdadeiro ou falso: (Q1 - falsa) a possibilidade de ocorrência de hipertensão arterial sistêmica e diabete mellitus associadas, em um mesmo paciente, é rara; (Q2 - verdadeira) são atribuições da equipe do Posto de Saúde na prevenção de hipertensão arterial sistêmica e diabetes mellitus realizar campanhas educativas e programar, periodicamente, atividades de lazer individual e comunitário; (Q3 - falsa) antes de recomendar a prática de exercícios físicos, o médico deve solicitar um eletrocardiograma; (Q4 - verdadeira) o tratamento inicial do indivíduo obeso visa promover a perda de $5 \%$ a $10 \%$ do peso inicial em até 6 meses; (Q5 - falsa) o consumo de frutas, verduras e legumes deve ser restringido a 5 porções/dia (400-500g); (Q6 - falsa) geralmente, o problema do alcoolismo é de instalação abrupta, em decorrência de algum fato marcante (luto, desemprego etc.); (Q7 - verdadeira) o simples aconselhamento de parar de fumar possui benefício comprovado para efetivo abandono do tabagismo 9,10.

O desempenho dos médicos foi quantificado mediante cálculo de três médias: teórica, prática e geral (média entre teórica e prática). A média teórica foi calculada dividindo-se o número de acertos de cada médico pelo total de questões (sete), multiplicado por dez. Cada médico recebeu uma nota que podia variar de zero a dez. Foram considerados com nível de conhecimento "ótimo" os médicos que obtiveram mais de $70 \%$ de acertos; "bom”, os que acertaram 50\% a 70\% dos itens investigados; "regular", de 40\% a 49\%; e "ruim", quando inferior a $40 \%$ de acertos.

O desempenho prático foi avaliado dividindo-se por nove o número de condutas preventivas realizadas em cada consulta (investigação ou recomendação sobre fatores de risco para DCNT). O resultado foi multiplicado por dez. O cálculo foi ponderado pelo número de consultas de cada médico. A nota prática poderia variar de zero a dez. Para as análises, a nota prática foi estratificada conforme a queixa principal da consulta (DCNT e não DCNT). A média geral do desempenho dos médicos foi obtida pela soma das médias teórica e prática e dividindo-se por dois. Os médicos que obtiveram um desempenho com, no mínimo, nota cinco, tiveram um desempenho considerado "bom".
O controle de qualidade foi realizado por contato telefônico com os médicos, para confirmar a realização das observações das consultas durante todo o turno de atendimento, bem como para verificar a repetição de algumas respostas nos questionários autoaplicados. A digitação dos dados foi feita no programa Epi Info 6.04 (Centers for Disease Control and Prevention, Atlanta, Estados Unidos) e as análises no programa Stata 9.0 (Stata Corp., College Station, Estados Unidos).

Foram realizadas análises descritivas do conhecimento e desempenho dos médicos. Utilizou-se teste qui-quadrado para as análises bivariadas, com nível de significância de 5\%, bicaudal. Adicionalmente, comparou-se o desempenho conforme o tipo de UBS (ESF ou tradicionais). Para essa comparação foram calculadas as frequências das recomendações e itens do exame físico e seus respectivos intervalos de 95\% de confiança (IC95\%). Como se esperava uma agregação natural dos dados (pacientes consultando com o mesmo médico receberiam recomendações semelhantes e médicos de uma mesma UBS seriam mais parecidos entre si do que de outras UBS), realizou-se análise por multiníveis. Para ela, os dados foram organizados em três níveis: das UBS, dos médicos e dos pacientes. 11

O projeto de pesquisa foi aprovado pelo Comitê de Ética em Pesquisa da Universidade Federal de Pelotas sob o registro $n^{\circ}$. 093/06.

\section{Resultados}

A primeira etapa do estudo ocorreu entre setembro e dezembro de 2006, quando foram visitadas 28 UBS onde atuavam 61 médicos, sendo observadas 422 consultas. A segunda etapa ocorreu nos meses de maio e junho de 2007, quando foram entrevistados 59 médicos (duas perdas).

A maioria dos médicos era mulher $(n=42)$, com média etária de 47 anos. A Tabela 1 mostra as condições de saúde, formação acadêmica, características de trabalho na UBS (carga horária, tempo de atuação, satisfação e dificuldades). A maioria graduou-se em Pelotas há mais de 17 anos. Menos de um terço dos médicos $(n=16)$ possuía pós-graduação em saúde coletiva, os demais eram especialistas em outra área ou não haviam feito pós-graduação. A maioria trabalhava em UBS há mais de dez anos. Cerca da metade dos médicos estava satisfeita com o trabalho, embora a maioria estivesse insatisfeita com o salário percebido. A principal dificuldade apontada pelos médicos para o trabalho na UBS foi a falta de medicamentos para serem distribuídos aos usuários. $\mathrm{O}$ desconhecimento quanto à própria saúde foi baixo. Dois médicos desconheciam seu 
Perfil de saúde e formação acadêmica dos médicos da Atenção Primária à Saúde, características de trabalho, satisfação e dificuldades. Pelotas, Rio Grande do Sul, Brasil, 2006-2007 ( $n=59)$.

\begin{tabular}{|c|c|c|}
\hline & $\mathrm{n}$ & $\%$ \\
\hline \multicolumn{3}{|l|}{ Condição de saúde } \\
\hline Tabagismo & 7 & 11,9 \\
\hline Excesso de peso * & 26 & 44,1 \\
\hline Hipertensão & 14 & 23,7 \\
\hline Dislipidemia & 8 & 13,6 \\
\hline Diabetes & 2 & 3,4 \\
\hline Sedentarismo & 13 & 22,0 \\
\hline \multicolumn{3}{|l|}{ Formação acadêmica } \\
\hline \multicolumn{3}{|l|}{ Local de graduação } \\
\hline Pelotas & 50 & 84,7 \\
\hline Outro & 9 & 15,3 \\
\hline \multicolumn{3}{|c|}{ Tempo de formado (anos) } \\
\hline$<8$ & 6 & 10,2 \\
\hline $8-17$ & 18 & 30,5 \\
\hline $18-28$ & 22 & 37,3 \\
\hline$>28$ & 13 & 22,0 \\
\hline \multicolumn{3}{|l|}{ Pós-graduação } \\
\hline Não & 17 & 28,8 \\
\hline Saúde Coletiva & 16 & 27,1 \\
\hline Outras & 26 & 44,1 \\
\hline \multicolumn{3}{|c|}{ Características de trabalho } \\
\hline \multicolumn{3}{|c|}{ Carga horária semanal (horas) } \\
\hline$<20$ & 4 & 6,8 \\
\hline 20 & 21 & 35,6 \\
\hline $20-30$ & 9 & 15,3 \\
\hline 40 & 24 & 40,7 \\
\hline$>40$ & 1 & 1,7 \\
\hline \multicolumn{3}{|c|}{ Tempo em UBS (anos) } \\
\hline$\leq 5$ & 15 & 25,4 \\
\hline $6-10$ & 13 & 22,0 \\
\hline $11-20$ & 19 & 32,2 \\
\hline$\geq 21$ & 12 & 20,3 \\
\hline \multicolumn{3}{|l|}{ Satisfação } \\
\hline \multicolumn{3}{|l|}{ Com o trabalho } \\
\hline Insatisfeitos & 2 & 3,4 \\
\hline Pouco satisfeitos & 28 & 47,5 \\
\hline Satisfeitos & 25 & 42,4 \\
\hline Muito satisfeitos & 4 & 6,8 \\
\hline \multicolumn{3}{|l|}{ Com o salário } \\
\hline Insatisfeitos & 34 & 57,6 \\
\hline Pouco satisfeitos & 19 & 32,2 \\
\hline Satisfeitos & 4 & 6,8 \\
\hline Muito satisfeitos & 2 & 3,4 \\
\hline
\end{tabular}

(continua) 


\begin{tabular}{lcc}
\hline & $\mathbf{n}$ & $\%$ \\
\hline Dificuldades & 56 & 94,9 \\
Falta medicamento & 21 & 35,6 \\
Má estrutura física da UBS & 19 & 32,2 \\
Reclamações da comunidade & 8 & 13,6 \\
Trabalho em equipe & 7 & 11,9 \\
Excesso de trabalho & 30 & 50,8 \\
Outras ** & &
\end{tabular}

UBS: unidade básica de saúde.

* $I M C \geq 25 \mathrm{~kg} / \mathrm{m}^{2}$. Seis desconheciam o peso e três a altura (apenas um desconhecia o peso e a altura);

** Inclui: baixos salários, falta de segurança e de material para procedimentos, dificuldades no acesso a exames

complementares e deficiência no sistema de referência a serviços especializados.

perfil lipídico e um deles também não sabia se era hipertenso. $\mathrm{O}$ excesso de peso foi o fator mais referido, seguido por hipertensão arterial e sedentarismo.

\section{Conhecimento, desempenho e barreiras para prevenção de DCNT}

A maioria respondeu corretamente às questões Q2 $(\mathrm{n}=53)$ e Q6 $(\mathrm{n}=49)$. Apenas 23 médicos responderam corretamente a questão Q3 e 14, à Q7. A média de número de acertos foi quatro, e apenas um médico acertou todas as perguntas e nenhum acertou menos que duas questões. $\mathrm{O}$ desempenho teórico dos médicos correspondeu à média 6,0 (IC95\%: 5,8-6,1), em uma escala de zero a dez. A maioria dos médicos relatou a utilização de livros técnicos, artigos científicos, páginas da Internet e congressos para atualizarem seus conhecimentos.

Entre as 422 consultas observadas, a maioria dos pacientes era mulher (72,3\%), com média de 52 anos de idade (DP $=17$ ) e cor da pele branca (70,6\%). Cada médico atendeu, em média, a sete pacientes adultos no turno observado. Nos extremos, três médicos atenderam a apenas dois pacientes e um, a 26 (este excluído para o cálculo da média de atendimentos). A duração das consultas foi, em média, de 11 minutos ( $\mathrm{DP}=7$ ): $21 \%$ das consultas duraram menos de cinco minutos e 11\%, 21 minutos ou mais. As DCNT foram motivo de 51 consultas (12,1\%; IC95\%: 9,0-15,2). Entre as não DCNT ( $\mathrm{n}=371$ ), $76,5 \%$ foram por enfermidades agudas e $23,5 \%$ para solicitações de atestados, exames ou outros documentos.

O desempenho dos médicos para prevenção de DCNT durante as consultas está apresentado na Tabela 2. Destaca-se o baixo número de consultas em que foram abordados aspectos preventivos para as DCNT. Em mais de um ter- ço das consultas por DCNT (37,3\%) e em quase dois terços $(60,9 \%)$ das por não DCNT, os médicos não fizeram nenhuma recomendação preventiva. Nenhum dos três itens do exame físico foi aferido em $11,8 \%$ dos pacientes em consulta por DCNT e em quase a metade $(48,8 \%$ ) dos que consultaram por não DCNT. Ainda, em 7,8\% das consultas por DCNT e 35\% das por não DCNT, os médicos não deram nenhuma recomendação preventiva de DCNT nem aferiram peso, altura ou pressão arterial.

Entre todas as recomendações, as mais frequentes foram para cessação do tabagismo, promoção da atividade física e perda de peso. A aferição de pressão arterial foi a medida mais frequente. Quando a queixa principal da consulta era uma DCNT, os médicos fizeram mais recomendações quanto à perda de peso, promoção da atividade física, diminuição do consumo de frituras/gorduras e sal, além de aferirem mais frequentemente a pressão arterial, comparativamente às motivadas por queixas de não DCNT. A prevenção primária, entre os sem DCNT, foi feita apenas para pouco mais de um terço dos pacientes $(39,1 \%)$.

O desempenho dos médicos foi ainda analisado conforme o diagnóstico prévio de fatores de risco ou DCNT registrados no prontuário, independente do motivo da consulta atual (Tabela 3). Pacientes em primeira consulta ou com prontuário extraviado (19\%) foram considerados como não portadores dos fatores de risco ou DCNT. A hipertensão arterial foi o diagnóstico mais prevalente $(30,3 \%)$. Nestes, a conduta mais frequente foi a medida da pressão arterial (75\%) e a recomendação mais dada, com relação à dieta para perda de peso $(24,2 \%)$. Destaca-se o pequeno percentual de pacientes com diagnóstico registrado de hipertensão que receberam orientações quanto ao consumo de sal (13,3\%). 


\section{Tabela 2}

Recomendações sobre fatores de risco para doenças crônicas não transmissíveis (DCNT) e exame físico em todas as consultas e conforme o motivo. Pelotas, Rio Grande do Sul, Brasil, 2006-2007 ( $n=422$ ).

\begin{tabular}{|c|c|c|c|c|}
\hline \multirow[t]{2}{*}{ Variáveis } & \multirow[t]{2}{*}{$\%$} & \multicolumn{2}{|c|}{ Motivo da consulta (\%) } & \multirow[t]{2}{*}{ Valor de $\mathrm{p}$} \\
\hline & & $\begin{array}{c}\text { DCNT } \\
(n=51)\end{array}$ & $\begin{array}{c}\text { Não DCNT } \\
(n=371)\end{array}$ & \\
\hline \multicolumn{5}{|l|}{ Recomendações } \\
\hline Consumo de sal & 9,0 & 21,6 & 7,3 & 0,002 * \\
\hline Consumo de fritura/gordura & 11,6 & 23,5 & 10,0 & 0,005 \\
\hline Consumo de frutas/verduras & 7,6 & 9,8 & 7,3 & 0,7 * \\
\hline Perda de peso & 13,0 & 31,4 & 10,5 & $<0,001$ * \\
\hline Atividade física & 13,7 & 25,5 & 12,1 & 0,009 \\
\hline Tabagismo & 15,6 & 19,6 & 15,1 & 0,4 \\
\hline Nenhuma & 58,1 & 37,3 & 60,9 & 0,001 \\
\hline \multicolumn{5}{|l|}{ Exame físico } \\
\hline Aferição da pressão arterial & 55,5 & 88,2 & 50,9 & $<0,001$ \\
\hline Aferição do peso & 8,3 & 15,7 & 7,3 & 0,08 * \\
\hline Aferição da altura & 1,9 & 0,0 & 2,2 & - \\
\hline Nenhum dos três & 44,3 & 11,8 & 48,8 & $<0,001$ \\
\hline
\end{tabular}

* Qui-quadrado com correção de Yates.

Tabela 3

Recomendações sobre fatores de risco para doenças crônicas não transmissíveis (DCNT) e exame físico, conforme registro prévio de DCNT no prontuário no último ano. Pelotas, Rio Grande do Sul, Brasil, 2006-2007 ( $n=422$ ).

\begin{tabular}{|c|c|c|c|c|c|c|c|c|c|c|}
\hline \multirow{2}{*}{$\begin{array}{l}\text { Diagnóstico } \\
\text { registrado no } \\
\text { prontuário no ano } \\
\text { anterior à consulta }\end{array}$} & \multirow{2}{*}{$\begin{array}{c}\text { Prevalência } \\
\text { (\%) }\end{array}$} & \multicolumn{9}{|c|}{ Recomendações e exame físico (\% de consultas) } \\
\hline & & $\begin{array}{c}\text { Diminuir o } \\
\text { consumo } \\
\text { de sal }\end{array}$ & $\begin{array}{c}\text { Consumo } \\
\text { de fritura/ } \\
\text { gordura }\end{array}$ & $\begin{array}{l}\text { Consumo } \\
\text { de frutas/ } \\
\text { verduras }\end{array}$ & $\begin{array}{l}\text { Perda de } \\
\text { peso }\end{array}$ & $\begin{array}{l}\text { Atividade } \\
\text { física }\end{array}$ & Tabagismo & $\begin{array}{l}\text { Aferição } \\
\qquad \text { da } \\
\text { pressão } \\
\text { arterial }\end{array}$ & $\begin{array}{l}\text { Aferição } \\
\text { do peso }\end{array}$ & $\begin{array}{l}\text { Aferição } \\
\text { da altura }\end{array}$ \\
\hline $\begin{array}{l}\text { Hipertensão arterial } \\
\text { sistêmica }\end{array}$ & 30,3 & 13,3 & 20,3 & 11,7 & 24,2 & 19,5 & 9,4 & 75,0 & 14,1 & 3,1 \\
\hline Diabetes mellitus & 8,8 & 5,4 & 10,8 & 18,9 & 40,5 & 13,5 & 8,1 & 67,6 & 16,2 & 2,7 \\
\hline Excesso de peso & 2,8 & 8,3 & 0,0 & 8,3 & 41,7 & 41,7 & 0,0 & 41,7 & 16,7 & 0,0 \\
\hline Dislipidemia & 9,5 & 5,0 & 22,5 & 17,5 & 35,0 & 32,5 & 7,5 & 57,5 & 7,5 & 0,0 \\
\hline Cardiopatia isquêmica & 3,1 & 15,4 & 7,7 & 7,7 & 0,0 & 15,4 & 15,4 & 92,3 & 15,4 & 0,0 \\
\hline $\begin{array}{l}\text { Insuficiência cardíaca } \\
\text { congestiva }\end{array}$ & 2,4 & 0,0 & 30,0 & 0,0 & 30,0 & 20,0 & 20,0 & 80,0 & 40,0 & 0,0 \\
\hline Tabagismo & 3,8 & 18,8 & 12,5 & 6,3 & 6,3 & 18,8 & 75,0 & 68,8 & 12,5 & 6,3 \\
\hline Sedentarismo & 1,0 & 25,0 & 0,0 & 25,0 & 50,0 & 100 & 25,0 & 50,0 & 0,0 & 0,0 \\
\hline Não DCNT * & 59,7 & 7,9 & 8,3 & 6,0 & 6,8 & 9,9 & 16,7 & 46,0 & 5,2 & 1,6 \\
\hline
\end{tabular}

* Pacientes em primeira consulta $(n=47)$ ou com prontuários extraviados $(n=34)$ foram incluídos nessa categoria. 
Verificou-se a frequência de aferição de pressão arterial, peso e altura, conforme a existência dessa informação nos registros do último ano. Somente $7,2 \%$ dos pacientes sem registro anterior, contra $31,6 \%$ daqueles com registro, tiveram o peso aferido na consulta observada $(\mathrm{p}<0,001)$. Quanto à medida pressão arterial, os valores correspondentes foram $49,1 \%$ versus $77,7 \%$, respectivamente, dos sem registro e com registro prévio ( $\mathrm{p}<0,001)$. Apenas $1,2 \%$ de todos os pacientes observados tinha registro de altura no prontuário. Entre os pacientes sem registro $(98,8 \%)$, apenas oito foram medidos.

O principal obstáculo ao aconselhamento para prevenção de DCNT apontado pelos médicos foi a expectativa de não adesão dos pacientes às recomendações. Seguiram-se a falta de tempo durante as consultas e falta de interesse dos pacientes. Tais motivos foram apontados por $47,5 \%$, $27,1 \%$ e $17 \%$ dos médicos, respectivamente.

\section{Desempenho conforme o modelo de atenção}

As nove unidades de saúde incluídas na ESF eram assistidas por 19 médicos que atenderam a $27 \%$ do total de consultas observadas. Os médicos da ESF atenderam por turno, em média, a 6,5 pacientes (IC95\%: 6,0-6,9), e a duração média das consultas foi de 14,1 minutos (IC95\%: 12,7$5,4)$. A média de atendimentos para os médicos de UBS tradicionais foi de 7,6 pacientes (IC95\%: 7,4-7,8), e cada consulta com duração média de 10,9 minutos (IC95\%: 10,2-11,7). Nesses cálculos foi excluído o médico que atendeu 26 pacientes (este de uma UBS tradicional). A análise do desempenho dos médicos conforme o tipo de UBS é apresentada na Tabela 4. Os pacientes atendidos na ESF receberam mais recomendações quanto ao consumo de frutas/verduras do que aqueles atendidos em UBS tradicionais. As demais recomendações e os itens do exame físico foram realizados de forma semelhante entre os médicos dos dois grupos, como evidenciado pela sobreposição dos intervalos de confiança. A análise por multiníveis conforme o tipo de UBS (primeiro nível) e o médico (segundo nível) mostrou resultados semelhantes.

\section{Avaliação global}

A Tabela 5 apresenta as médias obtidas com a avaliação prática e teórica dos médicos, conforme o modelo de assistência. Os médicos da ESF obtiveram todas as médias superiores às dos do modelo tradicional. A média geral (teórica + prática) dos médicos dos dois modelos foi maior naquelas consultas motivadas por uma DCNT. Médicos com pós-graduação em saúde coletiva obtiveram desempenho melhor nas consultas, embora sem significância estatística, com nota prática de 2,3 (IC95\%: 1,8-2,8), em comparação àqueles com formação em outras áreas ou sem pós-graduação, que obtiveram nota 1,7 (IC95\%: $1,4-2,0)$.

\section{Discussão}

A principal limitação deste estudo foi a utilização de um grupo de perguntas e itens de observação, cuja validade na avaliação da qualidade do cuidado médico não foi anteriormente testada. No entanto, os quesitos investigados na avaliação teórica foram baseados nas recomendações do Ministério da Saúde e em livro-texto específico de Atenção Primária à Saúde, largamente utilizado nas duas universidades onde a maioria dos médicos em serviço fez a sua graduação 9,10. Quanto à observação de consultas, o viés esperado é de os médicos terem demonstrado um desempenho superior à prática habitual. As observações, contudo, fornecem informações mais acuradas do que as obtidas por entrevistas com médicos, fora do contexto das consultas.

Diversos fatores podem interferir no desempenho médico no cenário da consulta, entre eles: o conhecimento técnico, a satisfação com o trabalho, a receptividade percebida às orientações dadas 12,13,14,15, além da própria formação acadêmica. A formação dos recursos humanos é um fator determinante da qualidade do cuidado oferecido à população ${ }^{8}$. A literatura aponta que os médicos formados na área de saúde coletiva adotavam mais atitudes preventivas nas consultas e dispensavam mais tempo abordando mudança de estilo de vida, comparados aos médicos de outras especialidades 13,16,17. O atual estudo verificou que pouco mais de $25 \%$ dos médicos possuía alguma formação em saúde coletiva e eles obtiveram um desempenho melhor nas consultas, indicando a necessidade de maior investimento na formação dos profissionais para atuarem nesse nível de atenção.

Quando se avaliou o desempenho médico propriamente dito nas consultas, foi identificada uma alta frequência de aferições de pressão arterial, especialmente naquelas motivadas por doenças cardiovasculares ou seus fatores risco (hipertensão arterial, diabetes e tabagismo). Entretanto, a abordagem de medidas comportamentais foi muito baixa. Entre os pacientes com registro de diagnóstico de hipertensão arterial no ano anterior à consulta, encontrou-se resultado semelhante. Embora a aferição da pressão arterial seja um componente importante do acompanhamento dos pacientes hipertensos, 
Recomendações sobre fatores de risco para doenças crônicas não transmissíveis (DCNT) e exame físico, conforme o tipo de unidade básica de saúde (UBS), independente do motivo da consulta atual. Pelotas, Rio Grande do Sul, Brasil, $2006-2007$ $(n=422)$.

\begin{tabular}{|c|c|c|}
\hline & \multicolumn{2}{|c|}{ Tipo de UBS [\% (IC95\%)] } \\
\hline & ESF & Tradicional \\
\hline \multicolumn{3}{|l|}{ Recomendação } \\
\hline Consumo de sal & $14,9(7,6-20,5)$ & $7,1(4,3-10,0)$ \\
\hline Consumo de fritura/gordura & $18,4(11,2-25,6)$ & $9,1(5,9-12,3)$ \\
\hline Consumo de frutas/verduras & $14,9(8,3-21,6)$ & $4,9(2,5-7,3)$ \\
\hline Perda de peso & $14,9(8,3-21,6)$ & $12,3(8,6-16,0)$ \\
\hline Realização de atividade física & $19,3(11,9-26,7)$ & $11,7(8,1-15,3)$ \\
\hline Tabagismo & $21,9(14,2-29,6)$ & $13,3(9,5-17,1)$ \\
\hline \multicolumn{3}{|l|}{ Exame físico } \\
\hline Aferição da pressão arterial & $61,4(52,3-70,1)$ & $53,2(47,6-58,9)$ \\
\hline Aferição do peso & $7,0(2,3-11,8)$ & $8,8(5,6-11,9)$ \\
\hline Aferição da altura & $3,5(0,1-6,9)$ & $1,3(0,0-2,6)$ \\
\hline
\end{tabular}

ESF: Estratégia Saúde da Família.

\section{Tabela 5}

Médias teórica e prática (ponderada por número de consultas observadas), conforme o modelo de assistência. Pelotas, Rio Grande do Sul, Brasil, 2006-2007 ( $n=59$ ).

\begin{tabular}{lccc}
\hline Médias & Geral & ESF & Tipo de UBS \\
& & $6,9(6,6-7,1)$ & $5,6(5,5-5,8)$ \\
\hline Teórica & $6,0(5,8-6,1)$ & $2,6(2,5-2,8)$ & $1,7(1,6-1,8)$ \\
Prática & $1,9(1,8-2,0)$ & $4,8(4,6-4,9)$ & $3,7(3,5-3,8)$ \\
Geral & $3,9(3,8-4,0)$ & $6,2(5,9-6,5)$ & $4,7(4,5-4,9)$ \\
DCNT * & $5,1(4,9-5,2)$ & $4,6(4,4-4,8)$ & $3,5(3,4-3,6)$ \\
Não DCNT * & $3,8(3,7-3,9)$ & & \\
\hline
\end{tabular}

DCNT: doença crônica não transmissível; ESF: Estratégia Saúde da Família; UBS: unidade básica de saúde.

* Média geral (teórica + prática) conforme a queixa principal da consulta.

é ineficaz no controle pressórico. Orientações quanto a medidas não farmacológicas deveriam fazer parte da rotina do atendimento desses pacientes ${ }^{18}$, porém as recomendações comportamentais foram dadas em uma pequena proporção das consultas observadas.

Os motivos apontados pelos médicos em Pelotas para tal constatação são consistentes com a literatura: a falta de tempo durante as consultas e o baixo interesse ou adesão dos pacientes às recomendações 12,13. Portanto, em 64\% dos casos, a explicação deslocou a falta de orientação para a esfera de responsabilidade do paciente. Uma alternativa para transpor tais dificuldades seria o incentivo ao trabalho multidisciplinar, em que diversos profissionais das UBS estivessem capacitados a orientar os pacientes quanto aos fatores de risco para DCNT, otimizando o tempo da consulta médica. Outra opção seria a realização de intervenções comunitárias veiculadas pelos próprios serviços de saúde ou outros canais da comunidade, buscando estimular a adoção de hábitos saudáveis de vida. Há ainda relatos de que estratégias de intervenções populacionais concebidas em parceria entre profissionais da saúde, gestores e a própria população-alvo têm demonstrado resultados animadores 1,4,5,19 e são recomendadas pela iniciativa CARMEN 3 . 
Quando se avalia o desempenho dos médicos e se traduz em uma nota, verifica-se que o desempenho teórico (média 6,0) foi bom. Já o desempenho prático, com média 1,9 , ruim. A baixa abordagem de modificação de hábitos de vida nas consultas, refletida na avaliação prática dos médicos pode ser ainda atribuída à falta de motivação. Grande parte dos médicos relatou diversas causas de insatisfação, como baixos salários, falta de medicamentos, demora na realização de exames complementares e consultas com especialistas, entre outras. A falta de tempo nas consultas, apontada como uma das principais barreiras para a prática preventiva, pode estar relacionada com a insatisfação salarial, visto que os profissionais trabalhariam em tempo reduzido para conciliar outro emprego e, assim, complementar sua renda.

Outra possível consequência da falta de motivação pode estar refletida no baixo número de consultas em que foi abordada a prevenção primária entre os pacientes não portadores de DCNT ou seus fatores de risco, já que esse tipo de conduta é uma das prioridades da Atenção Primária à Saúde. Os profissionais deveriam ser mais proativos na abordagem dessa população porque é alta a prevalência $(32,2 \%)$ de pelo menos um fator de risco (hipertensão arterial, diabetes, excesso de peso, tabagismo ou sedentarismo) entre adultos da cidade de Pelotas 20 e baixa a prevalência de consultas com DCNT como queixa principal $(12,1 \%)$. Tal conduta permitiria prevenir e/ou identificar fatores de risco mais precocemente.

Em relação aos itens de exame físico observados, os pacientes mais frequentemente examinados foram aqueles que já tinham algum registro no último ano. Ainda que a aferição da pressão arterial tenha sido o item do exame físico mais prevalente entre os pacientes que não tinham registro de hipertensão no prontuário, independente da idade, cerca da metade saiu da consulta sem essa medida. Tal achado indica que a busca do diagnóstico precoce das DCNT em indivíduos de maior risco também foi deficitária, o que está de acordo com outro estudo realizado em Pelotas para avaliar rastreamento de hipercolesterolemia. Neste, $27 \%$ dos indivíduos com indicação de rastreio não foram submetidos ao exame 21 .

Quando a avaliação de desempenho considerou o tipo de UBS (ESF e tradicional), verificou-se que os médicos da ESF apresentaram um ponto a mais nas médias teórica e prática do que os médicos das UBS tradicionais. Tendo em conta que a ESF propõe um contato mais próximo das equipes de saúde com a comunidade e uma postura proativa frente aos problemas de saúde-doença da população 22 , seria esperado que nesses serviços as ações preventivas fossem mais consistentes e sistemáticas. Os médicos vinculados à ESF atenderam, em média, a menos pacientes e suas consultas foram mais longas, quando comparadas às dos médicos das UBS tradicionais. Os achados são consistentes com os de um estudo realizado na Região Sul do Brasil, que demonstrou média de pacientes atendidos por hora menor nas UBS com ESF 23 . No mesmo estudo, os médicos da ESF recomendaram mais atividade física para adultos e idosos do que os médicos das UBS tradicionais, o que também se verificou no atual estudo, apesar da insignificância estatística.

Em vista do exposto, pode-se concluir que o cuidado médico na rede de Atenção Primária à Saúde em Pelotas foi insatisfatório quanto à prevenção primária das DCNT. Os médicos, apesar de demonstrarem um razoável conhecimento técnico, não os colocaram em prática adequadamente, focando mais a prevenção terciária do que a primária. Percebe-se que os serviços de saúde estão estruturados de forma a atender prioritariamente aos indivíduos com a doença já instalada ${ }^{10}$. Destacam-se como possíveis explicações a formação médica centrada nas especialidades e a falta de formação em saúde pública dos médicos de Atenção Primária à Saúde. A sociedade que recebe esse tipo de atendimento passa a entender o cuidado em saúde como prioritariamente curativo. Tal pensamento é reforçado pela maciça propaganda de medicamentos veiculada na mídia e nos consultórios médicos, tornando o cuidado em saúde cada vez mais "medicalizado".

A medicina preventiva envolve uma prática consciente de educação para a saúde e cabe ao médico, bem como aos demais profissionais da saúde, investigar os hábitos de vida e aspectos sociais de cada paciente, além de conhecer as medidas preventivas específicas, o custo de suas aplicações e a reação dos pacientes. O médico deve dar atenção especial aos pacientes em aparente estado de saúde e estender seus serviços preventivos, sempre que possível, a toda família, considerada como unidade social básica 24 . 


\section{Resumo}

Realizou-se um estudo transversal para avaliar o conhecimento e desempenho quanto à prevenção de fatores de risco para doenças crônicas não transmissíveis (DCNT) em adultos, dos médicos de Atenção Primária da rede urbana de Pelotas, Rio Grande do Sul, Brasil. Foram observadas 422 consultas realizadas por 61 médicos. Em 58,8\% das consultas por pacientes com DCNT e 25,3\% das por outros motivos, não foram feitas quaisquer recomendações preventivas nem foram aferidos peso, altura ou pressão arterial. Para os portadores de DCNT, as recomendações mais frequentes foram para perda de peso, promoção da atividade física e redução do consumo de gorduras e sal. Entre os não portadores de DCNT, somente $39,1 \%$ receberam recomendações de prevenção primária. O desempenho quanto à prevenção de DCNT foi ruim, contemplando mais a prevenção terciária (aconselhando os já portadores de DCNT) do que a primária (prevenindo a incidência de DCNT).

Doença Crônica; Atenção Primária à Saúde; Serviços de Saúde

\section{Colaboradores}

M. Capilheira conduziu a pesquisa, analisou os dados e redigiu a primeira versão do manuscrito. I. S. Santos revisou o manuscrito e introduziu modificações.

\section{Referências}

1. Korhonen T, Su S, Korhonen HJ, Uutela A, Puska P. Evaluation of a national Quit and Win contest: determinants for successful quitting. Prev Med 1997; 26:556-64.

2. Nissinen A, Berrios X, Puska P. Community-based noncommunicable disease intervention: lessons from developed countries for developing ones. Bull World Health Organ 2001; 79:963-70.

3. Pan American Health Organization. CARMEN - an iniciative for integrated prevention of noncommunicable diseases in the Americas. Washington DC: Pan American Health Organization; 2003.

4. Health Promotion Agency. CINDI Highlights n. 11; 2004. http://www.healthpromotionagency.org.uk/ Resources/corporate/cindi/pdfs/CINDI\%20High lights\%202004.pdf (acessado em 02/Jun/2009).

5. Puska P. Successful prevention of noncommunicable diseases: 25 years experiences with North Karelia Project in Finland. Public Health Med 2002; 4:5-7.
6. Ministério da Saúde. Guia metodológico de avaliação e definição de indicadores: doenças crônicas não transmissíveis e Rede Carmen. Brasília: Ministério da Saúde; 2007.

7. Santos IS, Victora CG. Serviços de saúde: epidemiologia, pesquisa e avaliação. Cad Saúde Pública 2004; 20 Suppl 2:337-41.

8. Donabedian A. The quality of care: how can it be assessed? JAMA 1988; 260:1743-8.

9. Ministério da Saúde. Plano de reorganização da atenção à hipertensão arterial e ao diabetes mellitus: manual de hipertensão arterial e diabetes mellitus. Brasília: Ministério da Saúde; 2001.

10. Duncan BB, Schimidt MI, Giugliani ERJ. Medicina ambulatorial: condutas de Atenção Primária baseada em evidências. 2a Ed. Porto Alegre: Editora Artmed; 2004

11. Goldstein H. Multilevel statistical models. 2nd Ed. London: Kendall's Library of Statistics; 1995. 
12. Bull FCL, Schipper ECC, Jamrozik K, Blanksby B. How can and do Australian doctors promote physical activity? Prev Med 1997; 26:866-73.

13. Cornuz J, Ghali WA, Di Carlantonio D, Pecoud A, Paccaud F. Physicians' attitudes towards prevention: importance of intervention-specific barriers and physicians' health habits. Fam Pract 2000;17:535-40.

14. Keller I, Legetic B. Training Chilean primary health care professionals in nutrition for noncommunicable disease prevention. Rev Panam Salud Pública 2004; 16:242-9.

15. Olivarius NDF, Palmvig B, Andreasen AH, Thorgensen JT, Hundrup C. An educational model for improving diet counseling in primary care. A case study of the creative use of doctors' own diet, their attitudes to it and to nutritional counseling of their patients with diabetes. Patient Educ Couns 2005; 58:199-202.

16. Franck E, Harvey LK. Prevention advice rates of women and men physicians. Arch Fam Med 1996; 5:215-9.

17. Barnsley J, Willians P, Cockerill R, Tanner J. Physician characteristics and the physician-patient relationship - impact of sex, year of graduation and specialty. Can Fam Physician 1999; 45:935-42.

18. Ministério da Saúde. Hipertensão arterial sistêmica para o Sistema Único de Saúde. Brasília: Ministério da Saúde; 2006.
19. U.S. Department of Health and Human Services. Worldwide efforts to improve heart health: a follow up to the Catalonia Declaration - Selected Program Descriptions. Atlanta: U.S. Department of Health and Human Services; 1993.

20. Capilheira MF, Santos IS, Azevedo MR, Reichert FF. Risk factors for chronic non-communicable diseases and the CARMEN initiative: a populationbased study in the south of the Brazil. Cad Saúde Pública 2008; 24:2767-74.

21. Duro LN, Assunção MC, Dias-da-Costa JS, Santos IS. Desempenho da solicitação do perfil lipídico entre os setores público e privado. Rev Saúde Pública 2008; 42:82-8.

22. Ministério da Saúde. Política nacional de atenção básica. 4a Ed. Brasília: Ministério da Saúde; 2007.

23. Facchini LA, Piccini RX, Tomasi E, Thumé E, Silveira DS, Siqueira FV, et al. Desempenho do PSF no sul e no nordeste do Brasil: avaliação institucional e epidemiológica da Atenção Básica à Saúde. Ciênc Saúde Coletiva 2006; 11:669-81.

24. Arouca S. O dilema preventivista: contribuição para a compreensão e crítica da medicina preventiva. Rio de Janeiro: Editora Fiocruz; 2003.

Recebido em 22/Fev/2010

Versão final reapresentada em 15/Fev/2011

Aprovado em 26/Abr/2011 